gekennzeichnet - mit den bekannten Folgen für die Regierungsbildung. Was die Hintergründe des Wahlverhaltens betrifft, so waren es weniger die von den Parteien lancierten Themen (Jugend-)Kriminalität und Mindestlohn, die den Wahlausgang beeinflussten, sondern die Bildungspolitik: Die Positionen der Union in diesem Bereich bescherten ihr massive Stimmenverluste, von denen die SPD profitieren konnte.

(6) Das Wahlergebnis brachte eine Neuauflage der „hessischen Verhältnisse“: Ebenso wie 1982 und 1983 führte die erstmalige Repräsentanz einer neuen Partei im Landesparlament - damals der Grünen, jetzt der Linkspartei - zu einer Sitzverteilung, auf deren Grundlage keine mehrheitsfähige Regierung gebildet werden konnte. Die traditionelle Polarisierung der hessischen Politik schloss eine Große Koalition aus, lagerübergreifende Dreierverbindungen kamen ebenfalls nicht zustande. Den Regeln der Hessischen Landesverfassung entsprechend blieb daher die amtierende CDU-Alleinregierung geschäftsführend im Amt. In dieser Situation entschied sich die SPD für den Versuch, trotz ihrer klaren Ablehnung eines solchen Bündnisses im Wahlkampf eine Kooperation mit der Linkspartei anzustreben. Zwei Versuche, eine von der Linkspartei tolerierte rot-grüne Regierung zu bilden, scheiterten jedoch schon im Vorfeld, weil einzelne Abgeordnete der SPD diesem Vorhaben die Gefolgschaft verweigerten. Nach der endgültigen Implosion dieses Projektes blieb nur noch der Weg zu Neuwahlen.

\title{
Die bayerische Landtagswahl vom 28. September 2008: Betriebsunfall oder Ende eines Mythos?
}

\author{
Rainer-Olaf Schultze unter Mitarbeit von Jan Grasnick
}

Bei der Wahl am 28. September 2008 verlor die christlich-soziale Regierungspartei im Vergleich zur vorangegangenen Landtagswahl 17,3 Prozentpunkte. Sie blieb mit 43,4 Prozent der Gesamtstimmen deutlich hinter dem selbst gesteckten Wahlziel von 50 Prozent plus $\mathrm{x}$ zurück. Die absolute Zahl der Gesamtstimmen liegt bei etwa 4,6 Millionen und ist somit seit zehn Jahren bei Landtagswahlen rückläufig. Die CSU büßte dabei ihre - 2003 allerdings unter besonderen Bedingungen gewonnene - Zweidrittelmehrheit der Mandate im Maximilianeum ein und ist - jetzt auch in Bayern - im Fünf-Fraktionen-Parlament erstmals seit 46 Jahren auf einen Koalitionspartner angewiesen. ${ }^{1}$

Der bayerische Wähler löste damit ganz sicher einen Erdrutsch mit vielfältigen und tiefgehenden Konsequenzen aus; doch ist damit die Frage noch nicht beantwortet, ob es sich

1 Die wahlstatistische Dokumentation des Beitrags beruht auf der offiziellen Veröffentlichung des Bayerischen Landesamtes für Statistik und Datenverarbeitung, Wahl zum Bayerischen Landtag am 28. September 2008. Endgültiges Ergebnis, München 2008. Außerdem wurden Daten von den Webseiten des Landesamtes entnommen (www.statistik.bayern.de). Der wahlsoziologischen Interpretation liegen zugrunde: Infratest dimap, Wahlreport. Landtagswahl in Bayern am 28. September 2008, Berlin 2008; Forschungsgruppe Wahlen (FGW), Wahl in Bayern. Eine Analyse der Landtagswahl vom 28. September 2008, Mannheim 2008; Auswertungen der Süddeutschen Zeitung und des Münchner Merkurs. 
dabei „nur“ um einen der konkreten Situation geschuldeten und vergleichsweise leicht korrigierbaren Ausrutscher gehandelt hat, oder ob das Wahlergebnis vom 28. September das Ende des selbststilisierten Mythos von der natürlichen Vorherrschaft der CSU in Bayern signalisiert.

In Wahlen kristallisieren sich bekanntlich individuelle Präferenzen zu kollektiven Entscheidungen. Wählerentscheidungen sind komplex - gebildet aus kurzfristigen Einflussfaktoren, politisch-konjunkturellen und politisch-situativen wie aus dauerhaften strukturellen Determinanten und deren längerfristig wirksamen Veränderungen. Die Wahlforschung konstatiert dabei seit langem im bundesrepublikanischen Politikprozess nachhaltige Wandlungstendenzen. Diese finden zum einen auf der Angebotsseite des Wählermarktes statt und bestehen konkret aus tief greifenden Bindungsverlusten und Performanzproblemen der Volksparteien, verbunden mit der Fragmentierung des Parteiensystems und der Pluralisierung des Parteienangebotes. Zum anderen wandeln sich auch politische Partizipationsmuster der Wähler - insbesondere die generellen Trends wachsender Individualisierung und steigender Volatilität - bei allerdings noch immer strukturierter Vielfalt der Wählerentscheidung. ${ }^{2}$

$\mathrm{Zu}$ fragen ist also, in welchem Verhältnis die eher situativen Kurzzeitfaktoren zu den langfristigen Veränderungstendenzen im Wählerverhalten stehen, um sich auf diese Weise möglichen Antworten auf die eingangs gestellte Leitfrage zu nähern.

\section{Ausgangslage und Wahlkampf}

Nach der Landtagswahl von 2003 verfügten Edmund Stoiber und die CSU über eine komfortable verfassungsändernde Mandatsmehrheit im Landtag, die allerdings zu einem Gutteil den besonderen bundespolitischen Umständen der Jahre 2002/2003 geschuldet war: der offenkundigen „Mir-san-mir-Reaktion“ vieler Bayern nach der knappen Wahlniederlage Edmund Stoibers bei seiner Kanzlerkandidatur gegen Gerhard Schröder 2002 und dem Ansehensverlust vor allem der Sozialdemokratie im Gefolge der „Agenda 2010“-Politik der rot-grünen Bundesregierung ab Frühjahr 2003. Die deutlichen relativen Zugewinne verdeckten zudem den Tatbestand, dass die CSU bereits 2003 - wenn auch noch geringfügige - Stimmenverluste hatte hinnehmen müssen und dennoch ihren Stimmenanteil auf über 60 Prozent verbessern konnte. ${ }^{3}$ Die Zweidrittelmandatsmehrheit verleitete die Union und insbesondere ihr Führungspersonal zur Hybris - sach- wie personalpolitisch. Sachpolitisch ordnete die Regierung Stoiber ihre zentralen Vorhaben der Politik der Haushaltskonsolidierung und dem Ziel des ausgeglichenen Etats unter. Die „Politik des Rotstifts“ führte in den verschiedensten Politikfeldern zu Reformergebnissen, die an den Bedürfnissen und Interessen weiter Kreise der Bevölkerung vorbeigingen, auf deren Kritik man so gut wie keine

2 Vgl. Rainer-Olaf Schultze, Strukturierte Vielfalt als Wählerentscheidung heute? Eine Analyse der Bundestagswahl vom 22. September 2002, in: Bayerische Landeszentrale für politische Bildungsarbeit, Bilanz der Bundestagswahl 2002. Voraussetzungen, Ergebnisse, Folgen, München 2003, S. 71 - 100; Jörg Broschek / Rainer-Olaf Schultze, Wahlverhalten: Wer wählt wen?, in: Beate Hoecker (Hrsg.), Politische Partizipation zwischen Konvention und Protest. Eine studienorientierte Einführung, Opladen 2006, S. $23-54$.

3 Siehe zur vorangegangenen Landtagswahl Günter Rieger, Die bayerische Landtagswahl vom 21. September 2003: Banale Sensationen, in: ZParl, 34. Jg. (2003), H. 4, S. $702-720$. 
Rücksicht nahm; zudem waren manche der Vorhaben von handwerklichen Fehlern gekennzeichnet. Exemplarische Beispiele waren die vom damaligen Chef der Staatskanzlei Erwin Huber verantwortete umfassende Verwaltungsreform ${ }^{4}$, einschließlich der Erhöhung der Wochenarbeitszeit für Beamte, des Stellenabbaus im Öffentlichen Dienst, selbst bei der Polizei, sowie insbesondere die von den Kultusministern Monika Hohlmeier und Siegfried Schneider verantwortete Schulpolitik des G8-Gymnasiums. Der Unmut von Schülern, Lehrern und Eltern resultierte auch daher, dass mit der Reduzierung der Schulzeit auf acht Jahre gleichzeitig ein neuer Lehrplan für das alte neunjährige Modell erschien, der noch auf neun Jahre ausgelegt war und nun in der verkürzten Zeit zu bewältigen vorgesehen war. ${ }^{5}$ Die weit verbreitete Unzufriedenheit mit der Regierungspolitik kontrastierte dabei auffallend mit der demoskopisch regelmäßig gemessenen hohen Kompetenzzuweisung der Wähler auf dem Felde der Wirtschaftspolitik. Der Unmut gründete sich damit sowohl auf die konkreten Inhalte der weiteren wichtigen Felder der Landespolitik als auch auf den Regierungsstil und insbesondere auf das eingeschlagene Tempo bei der Umsetzung der haushaltspolitisch induzierten Reformagenda.

Beschleunigt wurde der Vertrauensverlust in die Handlungsfähigkeit der Regierung Stoiber durch eine Reihe kleinerer und größerer Skandale, beispielsweise im Lebensmittelund Umweltbereich oder die innerparteilichen Münchener Mitgliedschaftsskandale, in deren Gefolge Monika Hohlmeier von Minister- und Parteiamt zurücktreten musste ${ }^{6}$, vor allem aber durch das „Hin-und-Zurück“ Edmund Stoibers um seinen Eintritt als Superminister in die Berliner Große Koalition nach der Bundestagswahl von 2005, das ihm von vielen auch innerhalb der CSU-Mitgliedschaft als Flucht ausgelegt wurde. ${ }^{7}$ Stoiber leitete dadurch seinen Machtverfall selbst ein und beschädigte zugleich seine potentiellen Amtsnachfolger Günther Beckstein und Erwin Huber. ${ }^{8}$ Das Fass zum Überlaufen brachte die Affäre um die damalige Fürther Landrätin und CSU-Vorstandsmitglied Gabriele Pauli, als Stoiber deren öffentliche Kritik an seiner Amtsführung und ihren berechtigten Vorwurf, die Staatskanzlei überwache ihr Privatleben, mit dem Satz: „Sie sind nicht wichtig“ vom Tisch zu wischen versuchte. Die Affäre - eher Symptom als Ursache - führte Anfang 2007 zum von der CSU-Landtagsfraktion erzwungenen Amtsverzicht Stoibers und seiner Ablösung

4 Symptomatisch für die Abgehobenheit der Regierungspolitik, ja die Arroganz der Macht war etwa Erwin Hubers Satz: „Wer einen Teich trockenlegen will, der darf vorher nicht die Frösche fragen", mit der er die Kritik der Betroffenen des Öffentlichen Dienstes an den Verwaltungsreformvorhaben kommentierte, nach: SZ vom 14. Januar 2004, S. 3.

5 Exemplarisch Christine Burtscheidt, Druck auf Schulminister Schneider wächst, in: SZ vom 6. März 2008 sowie Birgit Taffertshofer, Aufstand der Verunsicherten, in: SZ vom 11. April 2008.

6 Zum Gammelfleisch-Skandal siehe die Beiträge in der SZ vom 29. August 2007, S. 33 und vom 24. April 2008, S. 50. Zur Hohlmeier-Wahlaffäre siehe SZ vom 16. Juli 2007, S. 53 und vom 27. Januar 2007, S. 43.

7 Zu den Konsequenzen siehe SZ vom 9. Dezember 2005, S. 4.

8 Für eine eingehende Analyse über den Verlauf und die Ursachen der Demontage Edmund Stoibers siehe Heinrich Oberreuter, Stoibers Sturz. Ein Beispiel für die Selbstgefährdung politischer Macht, in: ZParl, 39. Jg. (2008), H. 1, S. 112 - 118. Einen aufschlussreichen Vergleich zwischen Regierungen verschiedener Ebenen in westlichen Ländern hinsichtlich Machterwerb und -erosion (vor allem Kapitel II: Landesregierungen und Landesparteien - ausgewählte Fallbeispiele) liefern Gerhard Hirscher / Karl-Rudolf Korte (Hrsg.), Aufstieg und Fall von Regierungen. Machterwerb und Machterosionen in westlichen Demokratien, München 2001. 
durch das Tandem Beckstein/Huber mit Günther Beckstein als Ministerpräsidenten und Erwin Huber als CSU-Parteivorsitzendem. ${ }^{9}$ Allerdings gelang es beiden nicht, aus dem Schatten Stoibers herauszutreten. Zum einen gestatteten sie ihm, noch neun Monate bis zum September 2007 im Amt zu bleiben, so dass sie nur mehr ein Jahr Zeit hatten, sich selbst zu profilieren, die Partei zu konsolidieren und personell wie programmatisch in die Landtagswahl zu führen. Zweitens formulierten Stoiber und die Staatskanzlei während dieser Übergangszeit noch das so genannte Zukunftsprogramm „Bayern 2020“, so dass das Tandem Beckstein/Huber inhaltlich kaum eigene Akzente setzen konnte, sondern im Gegenteil beiden die Hände weitgehend gebunden waren.

Während sich die Stimmen- und Mandatsverluste der CSU bei den Wahlen der Jahre 2004/2005 noch in Grenzen hielten - bei der Europawahl 2004 erzielte die Partei bei einer Wahlbeteiligung von 39,7 Prozent 57,4 Prozent der Stimmen; bei der Bundestagswahl 2005 verfehlte sie mit 49,2 Prozent die magische 50-Prozent-Grenze nur knapp -, brach sie nach den Turbulenzen zum Jahreswechsel 2006/2007 und der fast einjährigen Agonie des Amtswechsels in Regierung und Partei bei den Kommunalwahlen im März 2008 dramatisch ein. ${ }^{10}$ Bei einer Wahlbeteiligung von unter 60 Prozent kam die CSU landesweit insgesamt nur auf rund 40 Prozent der Stimmen und verlor hauptsächlich an die kleinen Parteien, allen voran die Grünen und die Freien Wähler (FW). Letztere konnten zusammen 19 Prozent der Stimmen auf sich vereinigen. Die Kommunalwahlergebnisse waren damit ein Vorbote für die Ereignisse bei der Landtagswahl, denn von den offenkundigen Ansehensverlusten vermochten sich Partei- und Regierungsführung während des Wahlkampfes nicht mehr zu erholen. Im Gegenteil: Es verfestigte sich der Eindruck fehlender Handlungskompetenz - etwa auf dem Felde der Schul- und Bildungspolitik ${ }^{11}$, bei der Abwicklung des Transrapid-Projektes ${ }^{12}$, bei der Finanzkrise der Bayerischen Landesbank ${ }^{13}$-, und mangelnder Durchschlagskraft auf der Ebene der Bundespolitik - etwa in der Familien- und Sozial-

9 Erwin Huber wurde auf dem CSU-Parteitag vom 28. und 29. September 2007 zum neuen Parteivorsitzenden gewählt und setzte sich damit gegen seine Mitbewerber Horst Seehofer und Gabriele Pauli durch. Seehofer stellte zwar eine ernsthafte Konkurrenz gegenüber Huber dar, konnte sich aber in der Partei nicht durchsetzen. Günther Beckstein wurde am 9. Oktober 2007 vom Landtag zum Ministerpräsidenten gewählt.

10 Ergebnisse nach: Bayerisches Landesamt für Statistik und Datenverarbeitung (www.statistik.bayern.de); zur Einschätzung der Kommunalwahlergebnisse siehe Christian Deutschländer, Im Schlafwagen rumpelts, in: Online-Ausgabe des Münchner Merkurs (www.merkur-online.de, Abruf am 16. Mai 2008) beziehungsweise Katja Auer / Kassian Stroh, Alle sehen sich als Sieger, in: SZ vom 3. März 2008, S. 35.

11 Vgl. Infratest dimap, a.a.O. (Fn. 1), S. 27 und 30.

12 Das Transrapid-Projekt wurde von der Stoiber-Regierung gegen den Widerspruch weiter Bevölkerungskreise und der Münchener Stadtregierung stets massiv propagiert und gefördert; Stoiber und Huber feierten noch unmittelbar vor dem Amtswechsel die Sicherung der Finanzierung des Projektes, ehe es wenige Tage später von der neuen Regierung Beckstein wegen zusätzlicher Kosten und fehlender Finanzierung „beerdigt“ werden musste, siehe SZ vom 28. März 2008, S. 4.

13 Der Vorwurf der mangelnden Kontrolle der Bank durch die Politik traf vor allem Erwin Huber, der als Finanzminister im Verwaltungsrat des Unternehmens saß. Darüber hinaus wurde gegen ihn die Anschuldigung erhoben, über die tatsächliche Entwicklung der Verluste in der Bank der Öffentlichkeit zunächst nicht die Wahrheit gesagt zu haben, vgl. unter anderem die Berichte in der SZ vom 21. und 29. Mai sowie vom 19. September 2008. 
beziehungsweise Gesundheitspolitik ${ }^{14}$, in Sachen Pendlerpauschale und Steuersenkung ${ }^{15}$. Dort, wo die Regierung Beckstein - wie bei der strengen Nichtraucher-Regelung ${ }^{16}$ - aktiv wurde, oder bei verschiedenen für die nächste Wahlperiode ins Auge gefassten Infrastrukturvorhaben - wie in Sachen Ausbau des Münchener Flughafens oder der Donaukanalisierung - agierte sie neuerlich am Lebensgefühl und an den Interessen der Betroffenen vorbei, so dass der Eindruck von der Arroganz der Macht auch nach dem Amtswechsel fortbestand. Und dies umso mehr, als die CSU und ihr Spitzenpersonal an ihrer traditionellen Gleichsetzung der Partei mit Bayern, dokumentiert durch ein Wahlergebnis von deutlich über 50 Prozent der Stimmen, festhielten. Regierungschef Beckstein verstieg sich gar zu der Aussage: „Ein anständiger Bayer wählt CSU.“17

Auch in der SPD ereignete sich ein personeller Wechsel an der Spitze der Partei. 2004 wurde der Landespolitiker Wolfgang Hoderlein durch den Bundespolitiker Ludwig Stiegler als Landesvorsitzender abgelöst. Spitzenkandidat für die Landtagswahl wurde erneut der Pragmatiker Franz Maget, der Vorsitzende der SPD-Landtagsfraktion. Während des größten Teils der Wahlperiode konnten die Sozialdemokraten allerdings trotz umfänglicher Sacharbeit im Landtag kaum Akzente setzen, von denen sie wahlpolitisch bei den zahlreichen Wahlen nach 2003 und während des Wahlkampfes 2008 hätten profitieren können. So erreichten sie bei der Europawahl 2004 nur 15,2 Prozent, bei der Bundestagswahl 2005 immerhin 25,5 Prozent und mussten sich landesweit bei den Kommunalwahlen im Frühjahr 2008 mit 22,6 Prozent der Stimmen begnügen. Der offenkundige Unmut der Wählerschaft mit der CSU-Regierungspolitik wirkte sich auch während des Wahlkampfes nicht zugunsten der SPD aus - und dies, obwohl es dem Spitzenkandidaten Maget gelang, seinen Bekanntheitsgrad gegenüber der letzten Landtagswahl erheblich zu steigern und in einem direkten Fernsehduell mit dem Ministerpräsidenten Günther Beckstein einen kompetenten Eindruck zu hinterlassen. ${ }^{18}$

Die Schwerpunkte des Wahlkampfes lagen in den traditionellen Bereichen der sozialen Gerechtigkeit beziehungsweise gerechten Steuer- und Abgabenpolitik, mit denen die Partei jedoch nicht durchzudringen vermochte; zwar konnte die SPD ihre Kompetenzwerte auf diesen Themenfeldern deutlich verbessern, doch waren diese Themen für die bayerischen Wähler diesmal nur von nachrangiger Bedeutung und standen klar im Schatten etwa der Bildungs- und Schulpolitik. ${ }^{19}$ Zudem musste sich die SPD erstmalig jetzt auch in Bayern der Herausforderung von links stellen, so dass während des Wahlkampfes ein Hauptaugenmerk auf der Abwehr der Bedrohung durch die Partei Die Linke lag, der in Umfragen durchaus Chancen auf den Einzug in den Landtag eingeräumt wurden und die der SPD vor allem hinsichtlich des Themas der sozialen Gerechtigkeit ernsthaft Konkurrenz machte. Die Linke setzte dabei wie überall in der Republik mit ihrem Slogan „Hartz IV muss weg“ auf die Mobilisierung der Ängste weiter Bevölkerungskreise vor sozialem Abstieg beziehungsweise Verarmung, ohne jedoch in Anbetracht der vergleichsweise guten Wirtschafts-

14 Vgl. SZ vom 28. Mai 2008, S. 5.

15 Vgl. SZ vom 3. Juli 2008, S. 45.

16 Vgl. SZ vom 5. März 2008, S. 45 und vom 8. März 2008, S. 41.

17 So in einem Interview der Passauer Neuen Presse vom 2. August 2008.

18 Das Fernsehduell erfolgte am 18. September 2008 im Bayerischen Fernsehen.

$19 \mathrm{Vgl}$. Infratest dimap, a.a.O. (Fn. 1). Zur Interpretation siehe unter anderem SZ vom 27. August 2008, S. 33 und vom 4. Juli 2008. 
lage im Freistaat auf allzu große Resonanz zu stoßen. Zudem war ihr Spitzenkandidat, Fritz Schmalzbauer, in der Wählerschaft nahezu unbekannt. Trotz der offenkundigen Konkurrenzsituation auf dem linken Feld des Parteien- und Wählerspektrums führten die Sozialdemokraten jedoch keinen „Kreuzzug“ gegen die Linken, wie es Teile der CSU - mit Erwin Huber an der Spitze - unter heftiger Kritik eine Zeit lang als Strategie ausgaben. ${ }^{20}$ Hinderlich für Wahlkampf und Positionierung der Bayern-SPD waren dabei die Auseinandersetzungen in der Bundes-SPD kurz vor der Landtagswahl zum einen in der Frage der Koalitionsbildung mit der Linkspartei auf Landesebene, die vom Spitzenkandidaten Maget für Bayern zwar kategorisch ausgeschlossen worden war, die Partei im Wahlkampf aber dennoch in Argumentationsnöte brachte. Dies galt zum zweiten auch für die Querelen um den Wechsel im Bundesparteivorsitz, die trotz der überwiegenden Zustimmung zu Franz Müntefering gleichfalls den Wahlkampf der bayerischen Sozialdemokraten nachhaltig belasteten.

Mit neuen Doppelspitzen versuchte Bündnis 90/Die Grünen Akzente - nicht nur in Umweltfragen - in der Landespolitik zu setzen. Theresa Schopper und Sepp Daxenberger als Landesvorsitzende sowie Margarete Bause und Sepp Dürr als Fraktionsvorsitzende im Landtag führten die Politik der Partei in den gewohnten Bahnen, wobei Daxenberger als Spitzenkandidat ins Rennen ging. Mit ihm - ausgebildeter Landwirt und Schmied - gelang es den Grünen, auch die ländliche Bevölkerung verstärkt anzusprechen und gewisse „Berührungsängste“ abzubauen, etwa in Fragen der Nutzung gentechnisch veränderter landwirtschaftlicher Produktion, die von weiten Teilen gerade auch der ländlichen Bevölkerung kritisch gesehen wird. Hinzu kamen die Erfolge bei den vorangegangenen Wahlen - Europawahl 2004: 11,7 Prozent, Bundestagswahl 2005: 7,9 Prozent und Kommunalwahlen 2008: 8,2 Prozent der Stimmen. Die Grünen verstanden sich als „Premiumopposition“ ${ }^{21}$ und gingen infolgedessen zuversichtlich in den Wahlkampf. Ihnen kam dabei zugute, dass die Aufmerksamkeit gegenüber umwelt-, klima- und energiepolitischen Fragen in der Bevölkerung auch während des Wahlkampfes anhaltend hoch war. Dies galt ebenfalls für die Sorge um die Zukunft der Energieversorgung beziehungsweise den Streit um die Atomenergie ${ }^{22}$, da den Grünen auf diesen Feldern am ehesten die Lösung der Probleme zugetraut wurde. Weiter profitierten die Grünen offenbar davon, dass die Wirtschaftspolitik im Wahlkampf nicht im Vordergrund stand.

Spätestens seit der Kommunalwahl vom März/April befanden sich FDP und Freie Wähler deutlich im Aufwind. Den Freien Demokraten - seit 1982 nur einmal von 1990 bis 1994 im Landtag vertreten - gelang es dabei, auf innen- wie wirtschaftspolitischem Gebiet zu punkten. Ihre Landesvorsitzende Sabine Leutheusser-Schnarrenberger stand und steht für Grundrechtsschutz und Bürgerrechte, sichtbar unter anderem in der Verteidigung des Datenschutzes beziehungsweise im Eintreten gegen den Missbrauch von Daten von staatlicher und kommerzieller Seite sowie in der vehementen Kampagne gegen das reformierte Versammlungs- und Demonstrationsrecht. ${ }^{23}$ Der Spitzenkandidat Martin Zeil, Bundestagsabgeordneter und Generalsekretär der Landespartei, deckte im Wahlkampf die wirtschaftsli-

20 Vgl. SZ vom 27. August 2008, S. 33.

21 Vgl. SZ vom 7. Juni 2008, S. 50.

22 Während die CSU für eine Verlängerung der Laufzeiten der Atomkraftwerke plädierte, sprachen sich die Grünen, die SPD, die Freien Wähler und die Linke dagegen aus.

23 Neues Bayerisches Versammlungsgesetz vom 1. Oktober 2008. 
beralen und mittelstandsnahen Positionen ab, insbesondere auf dem Felde der Steuer(senkungs)politik, konkretisiert mit der Forderung nach Abschaffung der Erbschaftssteuer. Aufmerksamkeit erregte die FDP auch mit ihrer Ablehnung beziehungsweise wesentlichen Lockerung der strengen Nichtraucherschutzgesetzgebung der Staatsregierung. Die Freien Wähler - 2003 mit 4,0 Prozent der Stimmen an der Sperrklausel hängen geblieben, jedoch traditionell stark auf der kommunalen Ebene ${ }^{24}$ - forcierten seit dem Wechsel im Landesvorsitz von Armin Grein zum Niederbayern Hubert Aiwanger im Jahre 2006 ihre landespolitischen Ambitionen. Sie führten dabei den „Kampf“ um den ländlichen Raum sehr dezidiert, wobei die Ausgangsbasis ihre kommunalpolitische Repräsentation und Popularität, vor allem in den Gebieten, in denen sie den Landrat stellten, bildete. Zentrales Thema in ihrem Wahlkampf war ferner die Schul- und Bildungspolitik mit Forderungen nach der Reform des G8-Gymnasiums. Mit ihrer kritischen Einstellung zur Gentechnik versuchten die Freien Wähler auch in der Umweltpolitik zu punkten. Sie vertrauten zudem auf die Wirkung ihrer parteienkritischen Rhetorik, die sich in erster Linie gegen die CSU richtete. Beispielsweise machte eine nicht unerhebliche Zahl von ehemaligen CSU-Mitgliedern Wahlkampf für die Freien Wähler.

\begin{tabular}{|l|c|c|}
\hline Tabelle 1: Die wichtigsten politischen Probleme in Bayern vor der Landtagswahl \\
\hline & 2008 & 2003 \\
\hline Bildung / Schule / Ausbildung & 46 & 18 \\
\hline Arbeitslosigkeit / Arbeitsmarkt & 28 & 65 \\
\hline Wirtschaftliche Situation & 13 & 24 \\
\hline Familienpolitik / Kinderbetreuung & 11 & 6 \\
\hline Steuerbelastung / Mehrwertsteuer / Pendlerpauschale & 8 & 4 \\
\hline $\begin{array}{l}\text { Angaben in Prozent. Mehrfachnennungen möglich. Grundgesamtheit: Wahlberechtigte Bevölkerung in } \\
\text { Bayern. } \\
\text { Quelle: Infratest dimap, a.a.O. (Fn. 1), S. 25 f. }\end{array}$ \\
\hline
\end{tabular}

Anders als 2003 traf die regierende Union während des Wahlkampfes 2008 die Stimmung im Lande nicht, denn sie agierte weitgehend an den Themen, die einer Mehrheit der Wähler auf den Nägeln brannten, und an deren allerdings in den verschiedenen Landesteilen und Wählerschaften vielfältigen und auch gegensätzlichen Erwartungen vorbei. Zwar waren rund zwei Drittel der Wähler mit der wirtschaftlichen Situation im Lande zufrieden, doch spielte diesmal die kaum veränderte hohe Wirtschaftskompetenz der CSU in der Wählerentscheidung nur eine untergeordnete Rolle. An die Stelle von Wirtschaftsfragen und insbesondere der Arbeitslosigkeit trat als zentraler Gegenstand die Bildungspolitik (siehe Tabelle 1). Infratest dimap ermittelte, dass sie für 95 Prozent der Wähler bei ihrer Entscheidung von Bedeutung gewesen sei, gefolgt von Themen wie Atomenergie (84), Mindestlohn (76), Pendlerpauschale (67) oder die Probleme der Bayern LB (63 Prozent), während der Nichtraucherschutz und der Ausbau des Münchener Flughafens nur für Min-

24 Die Freien Wähler stellen 15 (darunter einen zusammen mit der CSU) von insgesamt 73 bayerischen Landräten, zum Beispiel in den Landkreisen Bad Tölz-Wolfratshausen, Freising und Nürnberger Land. 
derheiten relevant waren. ${ }^{25}$ Wichtiger als die Einschätzung der wirtschaftlichen Entwicklung war zudem, dass 60 Prozent aller Wähler und selbst 51 Prozent der CSU-Anhänger angaben, dass es in Deutschland eher ungerecht zugehe. Im Gegensatz zu 2003, als zwei Drittel der bayerischen Wähler mit der Politik der CSU-Regierung zufrieden gewesen waren, gab jetzt eine Mehrheit ein negatives Urteil über die Staatsregierung ab und fiel auch der Vorsprung des Amtsinhabers Beckstein vor dem Oppositionsführer Maget deutlich geringer aus als die Differenz in der Kandidatenalternative Stoiber - Maget bei der vorangegangenen Wahl.

Die schon bei den Kommunalwahlen sichtbar gewordene Wechselstimmung verstärkte sich in der Folgezeit und während der nur kurzen Phase des heißen Wahlkampfes nach Ende der Sommerferien - eine allerdings durchaus asymmetrische Wechselstimmung, von der nicht die parlamentarische Opposition von SPD und Grünen, sondern fast ausschließlich die außerparlamentarischen Liberalen und Freien Wähler profitierten. Rund zwei Drittel der Wähler gaben an, dass sie es begrüßen würden, wenn beide in den Landtag einziehen würden; nur mehr 36 Prozent votierten für eine CSU-Alleinregierung, während 60 Prozent sich für eine Koalitionsbildung unter Führung der Union und nur ganze 24 Prozent für eine Vierer-Koalition der Oppositionsparteien unter Führung von Franz Maget aussprachen. Die Präferenzen, welche der beiden kleinen bürgerlichen Parteien die CSU ins Regierungsboot nehmen sollte, waren mit 51 zu 49 zugunsten der FDP etwa gleich verteilt. Wie diese Daten aus der Wahltagbefragung von Infratest dimap signalisierten auch die Meinungsumfragen während des Wahlkampfes bereits das Ende der CSU-Alleinregierung. So konnte der Verlust der absoluten Mehrheit am Wahlabend nicht mehr überraschen, wenngleich die Stimmen- und Wählerverluste von rund 1,6 Millionen Gesamtstimmen gegenüber der Wahl von 2003 deftig ausfielen.

\section{Wablergebnis}

Zur Landtagswahl 2008 waren über 9,3 Millionen Bayern wahlberechtigt. Im Vergleich zu 2003 stieg die Wahlbeteiligung leicht von 57,1 auf 57,9 Prozent, bei (ausweislich der Daten der Repräsentativauszählung) allerdings deutlichen Unterschieden nach Alter und Geschlecht (siehe Tabelle 2). Gewinner nach Stimmen und Mandaten waren die „kleinen“ Oppositionsparteien, die zusammen deutlich über 30 Prozent der abgegebenen Stimmen auf sich vereinigten, insbesondere die beiden bis dato außerparlamentarischen Freien Wähler und Freien Demokraten (siehe Tabelle 3). Union und Sozialdemokraten hingegen kamen zusammen nur mehr auf 62,0 Prozent der Stimmen. Die CSU erlitt dabei dramatische Verluste (-17,3 Punkte auf 43,4 Prozent), und die SPD blieb mit 18,6 Prozent neuerlich unter der 20-Prozentmarke. Drittstärkste Kraft mit 10,2 Prozent und 21 Mandaten sind die Freien Wähler, die erstmals in den Bayerischen Landtag einzogen, gefolgt von den Grünen mit 9,4 Prozent und 19 Mandaten und der FDP mit 8,0 Prozent und 16 Mandaten. Die Linke, die erstmals antrat, scheiterte mit 4,4 Prozent an der Sperrklausel. Insgesamt entfielen immerhin 10,4 Prozent der Gesamtstimmen auf Parteien, die unter der Fünfprozenthürde blieben.

25 Vgl. Infratest dimap, a.a.O. (Fn. 1), S. 27. 
Tabelle 2: Wablbeteiligung nach Altersgruppen in Prozent

\begin{tabular}{|l|c|c|c|c|c|c|}
\hline & $\begin{array}{c}18-24 \\
\text { Jahre }\end{array}$ & $\begin{array}{c}25-34 \\
\text { Jahre }\end{array}$ & $\begin{array}{c}35-44 \\
\text { Jahre }\end{array}$ & $\begin{array}{c}45-59 \\
\text { Jahre }\end{array}$ & $\begin{array}{c}60+ \\
\text { Jahre }\end{array}$ & Zusammen \\
\hline Männer & 40,9 & 43,7 & 54,8 & 62,1 & 72,7 & 58,8 \\
\hline Frauen & 39,0 & 43,0 & 54,8 & 59,8 & 64,0 & 56,2 \\
\hline Männer und Frauen & 40,0 & 43,4 & 54,7 & 61,0 & 67,7 & 57,5 \\
\hline
\end{tabular}

Quelle: Bayerisches Landesamt für Statistik und Datenverarbeitung, Wahl zum Bayerischen Landtag am 28. September 2008. Repräsentative Wahlstatistik, München 2009.

\section{Tabelle 3: Amtliches Endergebnis der bayerischen Landtagswahl vom 28. September 2008}

\begin{tabular}{|l|r|r|r|r|c|c|}
\hline & \multicolumn{2}{|c|}{ Gesamtstimmen 2008 } & \multicolumn{2}{c|}{ Gesamtstimmen 2003 } & \multicolumn{2}{c|}{ Mandate 2008 } \\
\hline \multicolumn{1}{|c|}{ Partei } & Absolut & In Prozent & Absolut & In Prozent & Absolut & In Prozent \\
\hline CSU & 4.603 .960 & 43,4 & 6.217 .864 & 60,7 & 92 & 49,2 \\
SPD & 1.972 .437 & 18,6 & 2.012 .265 & 19,6 & 39 & 20,9 \\
Grüne & 999.111 & 9,4 & 793.050 & 7,7 & 19 & 10,2 \\
FW & 1.085 .896 & 10,2 & 411.306 & 4,0 & 21 & 11,2 \\
FDP & 847.227 & 8,0 & 263.731 & 2,6 & 16 & 8,6 \\
Die Linke & 461.755 & 4,4 & - & - & - & - \\
ödp & 212.200 & 2,0 & 200.103 & 2,0 & - & - \\
sonstige & 429.689 & 4,1 & 350.416 & 3,5 & - & - \\
\hline
\end{tabular}

Fehlende Übereinstimmungen zu 100 Prozent sind rundungsbedingt.

Quelle: Bayerisches Landesamt für Statistik und Datenverarbeitung, Wahl zum Bayerischen Landtag am 28. September 2008. Endgültiges Ergebnis, München 2008.

Dem Landtag gehören bei sieben Überhang- und Ausgleichsmandaten insgesamt 187 Abgeordnete an. Obwohl die CSU die absolute Stimmenmehrheit deutlich verfehlte, verfügt sie mit 92 Mandaten fast über die absolute Mehrheit der Sitze im Plenum des Landtages und über die Hälfte der Sitze in den Ausschüssen. Ursache für die starke Disproportion von Stimmen und Mandaten ist das bayerische Wahlsystem, die „verbesserte Verhältniswahl“, die im Vergleich zur übrigen Bundesrepublik eine Reihe von Besonderheiten aufweist ${ }^{26}$ : (1) Die lose gebundene Liste gibt den Wählern die Möglichkeit zur Präferenzstimmgebung mit der Zweitstimme; (2) Erst- und Zweitstimme werden beide bei der Stimmenverrechnung und Mandatszuteilung berücksichtigt; (3) die Stimmenübertragung erfolgt zwar auch nach dem Verfahren Hare/Niemeyer, allerdings nicht im gesamten Wahlgebiet, also landesweit/ gesamtbayerisch, sondern ausschließlich auf Wahlkreisebene in sieben Wahlkreisen unterschiedlicher Größ $\mathrm{e}^{27}$, die den Regierungsbezirken entsprechen und in denen insgesamt 180

26 Zum Wahlsystem siehe Emil Hübner, Das bayerische Landtagswahlrecht. Grundzüge - Nutzung - Reformmodell, in: Reinhold L. Bocklet (Hrsg.), Das Regierungssystem des Freistaats Bayern, Bd. 2, München 1979, S. 279 - 294; Rainer-Olaf Schultze / Jürgen Ender, Aus aktuellem Anlaß: Bayerns Wahlsystem - verfassungspolitisch bedenklich?, in: ZParl, 22. Jg. (1991), H. 1, S. 150 - 160; Gerhard Zeh, Benachteiligung kleiner Parteien durch das Bayerische Landeswahlgesetz, in: ZParl, 23. Jg. (1992), H. 2, S. $362-376$.

27 Die Zahl der (ohne Überhangmandate) 180 Mandate verteilt sich auf die Regierungsbezirke wie folgt: Oberbayern 58, Niederbayern 18, Oberpfalz 17, Oberfranken 17, Mittelfranken 24, Unterfranken 20, Schwaben 26 Mandate. In den Regierungsbezirken mit unter 20 Mandaten sind infolgedessen über fünf Prozent der Stimmen erforderlich, um dort an der Mandatsverteilung überhaupt beteiligt zu sein. 
Mandate vergeben werden (91 Direktmandate in den Stimmkreisen und 89 Mandate über Parteilisten in den Wahlkreisen). Zusammen mit der landesweiten Sperrklausel führt dies dazu, dass alle Parlamentsparteien profitieren, vor allem aber selbstverständlich die CSU am stärksten, wobei von der Sperrklausel die Hauptwirkung ausgeht. ${ }^{28}$

Das landesweite Ergebnis überdeckt dabei die regionalen Unterschiede, die diesmal zudem besonders ausgeprägt sind (siehe Tabelle 4). Zwar ist die Union überall stimmstärkste Partei geblieben, doch konnte sie in keinem der sieben bayerischen Bezirke eine absolute Mehrheit erringen. Die stärksten Einbußen musste sie mit 20,9 Prozentpunkten in Oberbayern hinnehmen. Hier erzielte sie mit nur mehr 39,3 Prozent den niedrigsten Anteil unter allen Bezirken. Stark überdurchschnittlich sind auch die Verluste in ihren weiteren traditionellen Hochburgen Niederbayern und der Oberpfalz: In Altbayern büßte sie 20,2 Prozentpunkte ein, während die Verluste in Franken mit 13,1 Punkten vergleichsweise moderat und mit 16,9 Punkten auch in Schwaben unterdurchschnittlich ausfielen. ${ }^{29}$ In diesen deutlichen Differenzen spiegeln sich zweifelsohne die nicht nur latent vorhandenen regionalen Animositäten, die durch den Wechsel an der Regierungsspitze vom katholischen Altbayern Stoiber zum protestantischen Franken Beckstein in starkem Maße aktualisiert worden waren und sind.

Die Sozialdemokraten erzielten ihre besten Ergebnisse mit knapp über 20 Prozent - von „Hochburgen“ wird man nicht sprechen können - weiterhin in Mittel- und Oberfranken, allerdings verloren sie hier auch am stärksten. Minimal zulegen konnte die SPD allein in Niederbayern, traditionell die Region mit den niedrigsten Stimmenzahlen. Überdurchschnittlich schnitt die SPD nur in Gebieten mit besonders hoher Bevölkerungsdichte ab, blieb allerdings selbst in München, Nürnberg oder Augsburg nur zweiter Sieger hinter der CSU, die in Gebieten mit hoher Bevölkerungsdichte allerdings unter 40 Prozent der Stimmen erreichte. Hingegen verbuchte die Union ihre besten Ergebnisse, wenngleich mit 47,1 Prozent auf deutlich niedrigerem Niveau, auch weiterhin auf dem Lande. ${ }^{30}$

Über regionale und lokale Schwerpunkte verfügen die drei „kleinen“ Parlamentsparteien. Sie sind im Vergleich zu den vorangegangenen Wahlen deutlich akzentuiert. Kerngebiet von Bündnis 90/Die Grünen ist Oberbayern, wo sie bei einem Zugewinn von 3,3 Punkten 13,1 Prozent der Stimmen gewannen. Erst mit großem Abstand folgen Mittelfranken, Schwaben und Unterfranken. Schwache Ergebnisse nur knapp über der Fünf-Prozent-Hürde erzielten sie in der Oberpfalz und in Niederbayern. Die Grünen sind auch weiterhin primär eine Partei (groß-)städtischer Wählerschaft, mit einem Stimmenanteil von 14,4 Prozent in München; überdurchschnittliche Zugewinne konnten sie aber auch in Gebieten mittlerer Bevölkerungsdichte verbuchen. Ähnliches gilt für die Liberalen, die den stärksten Wählerrückhalt in Oberbayern fanden (plus 7,0 auf 10,0 Prozent), gefolgt von Niederbayern und Schwaben. Die FDP verfügt über eine dezidiert urbane Klientel mit Wählern vor allem aus dem alten wie in geringerem Umfang aus dem neuen Mittelstand. In München kam die FDP immerhin auf 12,6 Prozent der Stimmen. Grüne und Liberale konkurrieren folglich regional und lokal gleichermaßen um städtische Wählerschaften, die sich allerdings

28 Bezogen auf die Zahl der verwerteten Gesamtstimmen erzielte die CSU einen Anteil von 48,4 Prozent und erhielt dafür 49,2 Prozent der Mandate.

29 Zur regionalen Stimmverteilung vgl. FGW, a.a.O. (Fn. 1), S. 70 ff.

30 Vgl. ebenda. In München erhielt die SPD beispielsweise 28,2 Prozent der Stimmen und liegt damit nur knapp hinter der CSU $(31,7)$. 


\begin{tabular}{|l|r|r|r|r|r|r|r|r|r|r|}
\hline Tabelle 4: Ergebnisse in den Regierungsbezirken und Veränderung gegenüber 2003 \\
\hline \multirow{4}{*}{ Bayern } & \multicolumn{2}{|c|}{ CSU } & \multicolumn{2}{|c|}{ SPD } & \multicolumn{2}{c|}{ Grüne } & \multicolumn{2}{c|}{ FW } & \multicolumn{2}{c|}{ FDP } \\
\cline { 2 - 12 } & $\%$ & Diff. & $\%$ & Diff. & $\%$ & Diff. & $\%$ & Diff. & $\%$ & Diff. \\
\cline { 2 - 11 } & 43,4 & $-17,3$ & 18,6 & $-1,0$ & 9,4 & $+1,7$ & 10,2 & $+6,5$ & 8,0 & $+5,4$ \\
\hline Bezirke: & & & & & & & & & & \\
Oberbayern & 39,3 & $-20,9$ & 19,3 & $-0,6$ & 13,1 & $+3,3$ & 8,8 & $+6,3$ & 10,0 & $+7,0$ \\
Niederbayern & 45,5 & $-19,4$ & 14,1 & $+0,0$ & 5,8 & $+1,4$ & 12,9 & $+6,7$ & 8,2 & $+6,2$ \\
Oberpfalz & 44,5 & $-18,1$ & 18,9 & $-0,4$ & 5,6 & $+0,6$ & 13,6 & $+8,4$ & 5,8 & $+4,1$ \\
Oberfranken & 44,7 & $-14,5$ & 21,2 & $-2,6$ & 6,7 & $+1,4$ & 10,7 & $+6,4$ & 6,6 & $+4,3$ \\
Mittelfranken & 43,7 & $-11,7$ & 22,6 & $-2,3$ & 8,7 & $+0,1$ & 9,3 & $+5,3$ & 6,1 & $+3,5$ \\
Unterfranken & 47,3 & $-13,3$ & 16,7 & $-1,7$ & 8,4 & $+0,8$ & 10,2 & $+5,6$ & 6,7 & $+3,9$ \\
Schwaben & 47,1 & $-16,9$ & 15,3 & $-0,5$ & 8,5 & $+1,0$ & 10,5 & $+5,7$ & 8,0 & $+5,6$ \\
\hline
\end{tabular}

Gesamtstimmenanteile in Prozent. Veränderungen zu 2003 in Prozentpunkten.

Quelle: Bayerisches Landesamt für Statistik und Datenverarbeitung, Wahl zum Bayerischen Landtag am

28. September 2008. Endgültiges Ergebnis, München 2008.

sozialstrukturell wie in Milieuverhaftung und Lebensstil voneinander unterscheiden.

Die Freien Wähler haben überproportional stark in eher ländlich geprägten Regionen abgeschnitten, und zwar vor allem dort, wo sie auf kommunaler Ebene bereits eine feste Basis besitzen. Die größten Zuwächse beziehungsweise Gesamtanteile weisen sie folglich in der Oberpfalz und in Niederbayern auf. Unterdurchschnittliche Anteile erzielten sie dagegen in Oberbayern und in Mittelfranken. Bayernweit verteilt sich der Erfolg der Freien Wähler dabei gleichermaßen auf Regionen mit niedriger (plus 7,2 auf 12,0 Prozent) und mittlerer Bevölkerungsdichte (plus 7,6 auf 12,2 Prozent), während sie in hoch verdichteten städtischen und großstädtischen Räumen nur auf 6,5 Prozent (plus 3,8) kamen und in München unter der Fünfprozenthürde blieben. ${ }^{31}$ Freie Wähler und Liberale konkurrieren damit regional und lokal um unterschiedliche Wählerschaften, die allerdings sozialstrukturell durchaus Ähnlichkeiten aufweisen.

\section{Zur wahlsoziologischen Struktur der Parteiwählerschaften}

\subsection{Die CSU - das Ende eines Mythos?}

Das besondere Markenzeichen der CSU war - verglichen mit ihrer Schwesterpartei CDU wie auch der anderen, der sozialdemokratischen Volkspartei - ihre in der Vergangenheit bei allen (Landtags-)Wahlen breite, ja dominante Repräsentation in so gut wie allen Wählerschichten und Milieus. Insofern kann es in Anbetracht des Gesamtergebnisses nicht überraschen, dass die Partei diesmal zweistellige Verluste in fast allen wahlsoziologisch relevanten Wählergruppen hat hinnehmen müssen. Allein bei den konfessionell-katholisch gebundenen und älteren Wählern konnte sie die Einbußen einigermaßen in Grenzen halten. Laut der Repräsentativauszählung nach Alter und Geschlecht erzielte die CSU nur mehr in der Altersgruppe der über 60-Jährigen über 50 Prozent der Stimmen (siehe Tabelle 5).

31 Vgl. ebenda. 


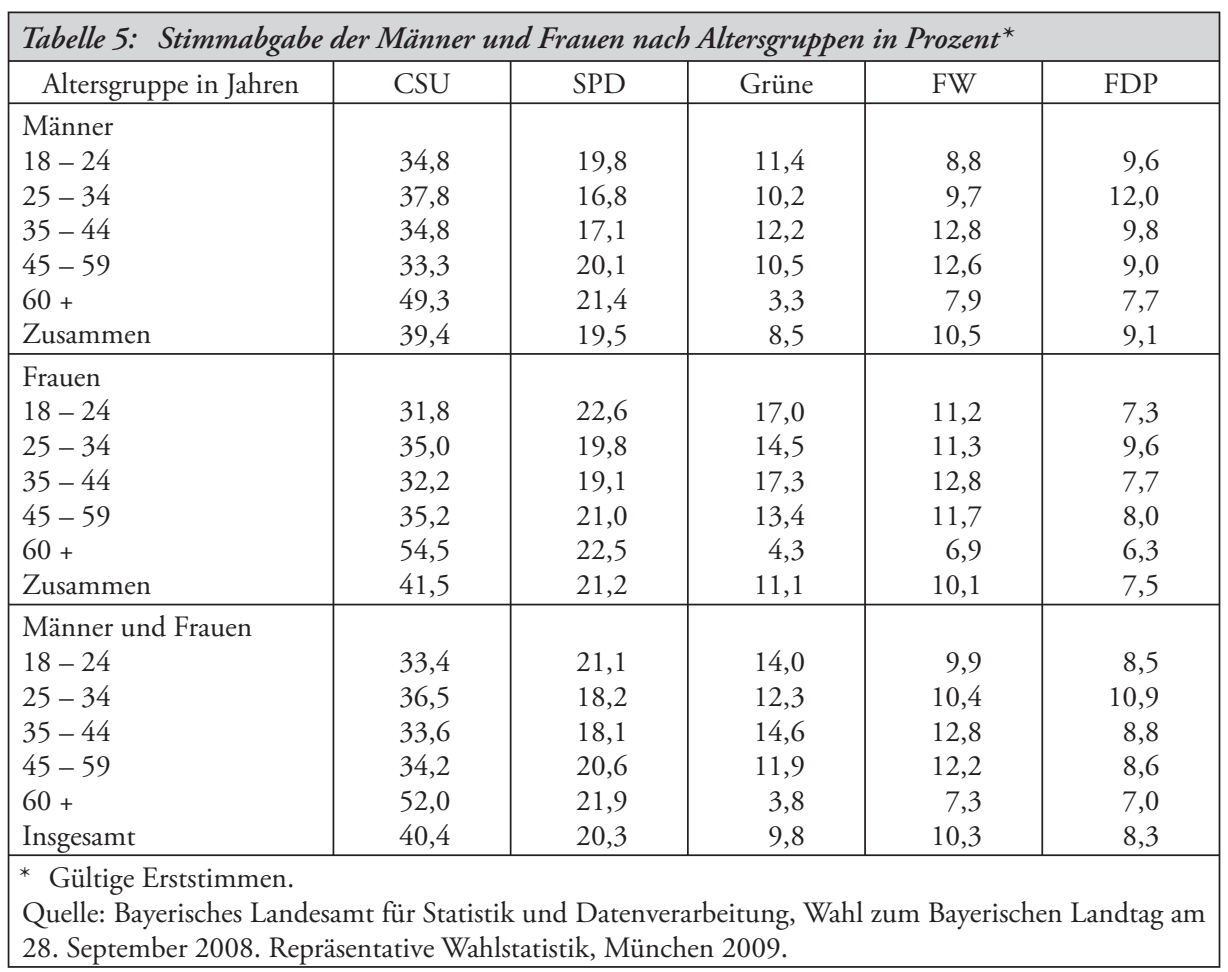

In den anderen Alterskohorten reichte es lediglich zu Stimmenanteilen zwischen 33 und 40 Prozent. Unterscheidet man nach Geschlecht, fallen die Verluste für die CSU bei den Frauen deutlich herber aus als bei den Männern. Bei den Erstwählerinnen in der Kohorte von 18 bis 24 Jahren summieren sich die Einbußen auf 22 Punkte, und die CSU erzielt nur noch knapp über 30 Prozent bei den Wählerinnen. Verstetigen sich diese Trends, sehen sich alle Parteien, aber insbesondere die CSU aufgrund ihrer ausgeprägten Unterrepräsentation in diesen Alterskohorten vor eine schwierige Situation und in Anbetracht der demographischen Entwicklung und der geringen Wahlbeteiligung in den Altersgruppen bis 35 Jahre vor ein strategisches Dilemma gestellt. Ihre Kernwählerschaft, deren Interessen und Bedürfnisse sie zu berücksichtigen hat, altert, während die nachrückenden Wähler, die es durch den entsprechenden Politikwandel zu gewinnen gilt, ihr den Rücken zukehren. Ähnlich verhält es sich im Blick auf den konfessionellen Faktor: Einerseits sind zwei Drittel aller CSU-Wähler katholisch; ihr bestes Ergebnis erzielte die CSU mit 69 Prozent bei den nur noch zwölf Prozent konfessionell-katholisch gebundenen Wählern, während sie bei allen Katholiken immerhin noch auf 49 Prozent der Stimmen kam. Andererseits lockert sich die konfessionelle Bindung insgesamt auch in Bayern deutlich, wächst die Gruppe der konfessionslosen Wähler und sind die Verluste der Union bei den Wählern mit mittlerer Bindung bei ca. 20 Prozentpunkten besonders hoch wie ihre Stimmenanteile bei den 12 Prozent Wählern ohne Konfession überdurchschnittlich niedrig. ${ }^{32}$ Auch hier ist in der Zukunft der 


\begin{tabular}{|c|c|c|c|c|c|c|c|c|c|c|}
\hline \multirow{3}{*}{ Bayern } & \multicolumn{2}{|c|}{ CSU } & \multicolumn{2}{|c|}{ SPD } & \multicolumn{2}{|c|}{ Grüne } & \multicolumn{2}{|c|}{$\mathrm{FW}$} & \multicolumn{2}{|c|}{ FDP } \\
\hline & $\%$ & Diff. & $\%$ & Diff. & $\%$ & Diff. & $\%$ & Diff. & $\%$ & Diff. \\
\hline & 43,4 & $-17,3$ & 18,6 & $-1,0$ & 9,4 & $+1,7$ & 10,2 & $+6,5$ & 8,0 & $+5,4$ \\
\hline $\begin{array}{l}\text { Bildung: } \\
\text { Hoch }\end{array}$ & 35 & -12 & 20 & -2 & 17 & +1 & 8 & +4 & 11 & +6 \\
\hline Mittel & 43 & -21 & 18 & -1 & 8 & +1 & 12 & +9 & 8 & +6 \\
\hline Niedrig & 51 & -16 & 19 & -1 & 3 & +1 & 11 & +6 & 6 & +5 \\
\hline $\begin{array}{l}\text { Beruf: } \\
\text { Arbeiter }\end{array}$ & 41 & -24 & 18 & +2 & 5 & +1 & 14 & +9 & 6 & +4 \\
\hline Angestellte & 36 & -21 & 20 & +1 & 14 & +3 & 12 & +7 & 8 & +6 \\
\hline Beamte & 31 & -11 & 24 & -5 & 15 & +1 & 13 & +10 & 9 & +5 \\
\hline Selbständige & 39 & -22 & 13 & -1 & 13 & +1 & 9 & +5 & 17 & +9 \\
\hline Landwirte & 52 & -39 & 0 & +0 & 3 & +3 & 26 & +17 & 11 & +11 \\
\hline Rentner & 56 & -8 & 21 & -5 & 4 & +1 & 6 & +4 & 6 & +5 \\
\hline In Ausbild. & 37 & -17 & 17 & -1 & 17 & +3 & 9 & +6 & 11 & +7 \\
\hline Arbeitslose & 35 & -19 & 27 & +15 & 7 & -6 & 5 & -1 & 3 & +1 \\
\hline
\end{tabular}

Gesamtstimmenanteile in Prozent. Veränderungen zu 2003 in Prozentpunkten. In der Kategorie Bildung ist hoch = Abitur / Fachhochschule / Universität; mittel = Mittel- / Realschule; niedrig = kein Abschluss / Volks- / Hauptschule.

Quelle: Infratest dimap, a.a.O. (Fn. 1), S. 47, S. 49.

Spagat zwischen konfessionell-katholischer Tradition und laizistischer Modernität gefordert, der von der Volkspartei CSU nur schwerlich und voraussichtlich nur unter beachtlichen Reibungsverlusten zu leisten sein dürfte.

Korreliert man die Wahlentscheidung mit Beruf und Bildung, zeigen sich beachtliche Varianzen im Umfang der CSU-Stimmenverluste (siehe Tabelle 6). Ihre stärksten Einbußen verzeichnete die CSU ausgerechnet bei ihrer ansonsten treuesten Wählerklientel. Lediglich 52 Prozent der Landwirte votierten für sie - ein Verlust von 39 Punkten.

Gleichfalls überdurchschnittlich fiel der Stimmenrückgang bei Arbeitern, Angestellten und Selbständigen aus. Die Union steht hier ganz offensichtlich in der Gefahr, ihre stets stolz betonte besondere Bindekraft einzubüßen, die eher sozialstaatsorientierte Arbeiter und Angestellte auf der einen Seite, eher liberal und freiheitlich orientierte Selbständige auf der anderen Seite unter einem Dach zu vereinigen wusste. Nur unterdurchschnittlich sind demgegenüber die Verluste bei Rentnern und Beamten. Ähnliche Differenzen bestehen beim Bildungsniveau der Wählerschaft, wobei der Stimmenanteil der CSU mit steigendem Bildungsniveau sinkt (siehe Tabelle 6). Hier zeigt sich ein weiteres Dilemma in der Vergangenheit erfolgreich regierender Volksparteien. Was die Sozialdemokraten beispielsweise in ihrem Stammland Nordrhein-Westfalen schon vor geraumer Zeit zu spüren bekamen, betrifft jetzt auch die bayerische CSU. Erfolgreiche Bildungspolitik und damit soziale Mobilität fördern die Lockerung beziehungsweise Aufösung traditioneller Milieus und tradierter Parteiidentifikationen, so dass sich mit gehobenem Bildungsniveau die Stammwählerschaft(en) der langjährig dominanten Volksparteien durchaus deutlich reduzieren und die Volatilität steigt. 


\subsection{FDP und Freie Wähler - die Gewinner der Wahl}

Die Stimmenverluste der CSU korrelieren in hohem Maße mit den starken Stimmenzuwächsen von FW und FDP - den eigentlichen Gewinnern der Wahl (siehe die Wanderungsbilanz in Abbildung 1). Wahlsoziologisch zeigen sich dabei überraschende Ähnlichkeiten wie zu erwartende Unterschiede in den Wählerschaften der beiden Konkurrenten der CSU im bürgerlichen Lager. Den Erwartungen entsprechen weithin die Unterschiede in der konfessionellen Zusammensetzung oder auch im Bildungsniveau der beiden Parteiwählerschaften. Bei den FW sind die konfessionell-katholischen Wähler (allerdings nur leicht) überrepräsentiert, während sie bei der FDP deutlicher unter- und die konfessionslosen Wähler beachtlich überrepräsentiert sind, denn die Freidemokraten erzielten bei letzteren Zuwächse von immerhin neun Punkten. ${ }^{33}$ Der Stimmenanteil der FDP steigt mit dem Bildungsniveau in der Wählerschaft; demgegenüber haben die FW überdurchschnittlich bei Wählern mit mittleren und niedrigen Bildungsabschlüssen abgeschnitten (siehe Tabelle 6). Im Blick auf die berufliche Zusammensetzung ihrer Wählerschaft erzielen die FW ihr bestes Ergebnis bei den Landwirten, gefolgt von Angehörigen selbständiger Berufe; eher unerwartet ist ihr gutes Abschneiden mit fast durchschnittlichem Ergebnis in der Arbeiterwählerschaft. Bei den Liberalen dominieren die Selbständigen, insbesondere aus dem alten Mittelstand, während ihre überdurchschnittlichen Erfolge bei Jungwählern in Ausbildung wie vor allem unter den Landwirten eher überraschen (siehe Tabelle 6). Altersmäßig sind die FW in den mittleren Kohorten zwischen 25 bis 60 Jahren am besten vertreten, die FDP hingegen bei den jüngeren bis 35 Jahren (siehe Tabelle 5). Thematische Kompetenzen und Schwerpunktsetzungen der beiden Parteien reflektieren dabei im Großen und Ganzen die sozialstrukturelle Basis ihrer Wählerschaften. Während die kritische Bewertung der CSUSchul- und Bildungspolitik durch die Wähler allen Oppositionsparteien zugute kam, profitierten die Liberalen speziell von den hohen Kompetenzzuweisungen ihrer Wähler auf den Feldern der Wirtschafts-, Steuer- und Abgabenpolitik, die FW demgegenüber von der ihnen zugerechneten sozialpolitischen Sachkompetenz vor allem in Fragen sozialer Gerechtigkeit und des Arbeitsmarktes. Allerdings waren sich die Wähler auch darüber im Klaren, dass die FW landespolitisch nichts werden verändern, aber frischen Wind in den Landtag bringen können. Einer solchen Frage stimmten drei Viertel der Wähler zu. ${ }^{34}$ Fraglich bleibt, ob sich auf derlei Motivationen Stabilität im Wählerverhalten gründen lässt.

\subsection{SPD und Grüne - Sachkompetenz ohne Wahlerfolg}

SPD und Grünen wiesen die Wähler ein beachtliches Maß an Sachkompetenzen zu, neben der Schul- und Bildungspolitik insbesondere auf ihren ureigensten Politikfeldern; den Grünen in der Umwelt-, Klima- und Energiepolitik, der SPD in Fragen sozialer Gerechtigkeit, der Arbeitsmarkt-, aber auch der Familien- und Wirtschaftspolitik. Beispielsweise waren kurz vor der Wahl 52 Prozent aller Wahlberechtigten der Meinung, dass die Grünen eine gute Umwelt- und Klimapolitik betreiben würden, und noch 30 Prozent trauten ihnen eine vernünftige Energiepolitik zu. Immerhin gaben deutlich über 30 Prozent der befragten 
Wahlberechtigten an, dass die Sozialdemokraten für soziale Gerechtigkeit einstehen, sich um die Sorgen der kleinen Leute kümmern und für eine gute Familienpolitik sorgen würden. Eine relative Mehrheit von 27 Prozent traute der SPD und damit mehr als allen anderen Parteien die Schaffung eines gerechten Steuer- und Abgabensystems zu. ${ }^{35}$ Themen- und Parteienkompetenz der Wähler fielen indessen bei beiden Parteien, vor allem aber bei den Sozialdemokraten, weit auseinander, so dass sich die positiven Sachkompetenzen nicht beziehungsweise nur in geringem Umfang in Stimmengewinnen niederschlugen, zumal nur rund ein Viertel der Wechselwähler lagerübergreifend wechselte.

Wahlsoziologisch zeigten sich in der grünen wie in der sozialdemokratischen Wählerschaft die bekannten Muster. Bündnis 90/Die Grünen erzielten ihre besten Ergebnisse erwartungsgemäß bei den Hochgebildeten sowie bei Erst- und Jungwählern mit Stimmenanteilen, die bei den Frauen rund ein Drittel über denjenigen der Männer liegen. Allerdings setzt sich das „Altern“ der Grünen Wählerschaft kontinuierlich fort, so dass die Partei mittlerweile in allen Alterskohorten bis zu 60 Jahren überdurchschnittliche Ergebnisse einfährt und nur bei den Wählern über 60 Jahren lediglich zwischen drei und vier Prozent erhielt (siehe Tabelle 5). Angestellte und Beamte, insbesondere aus den Berufen sozialer Dienstleistungen und der Wissensgesellschaft, sowie jugendliche Wähler in Ausbildung stellten auch weiterhin den Kern der grünen Wählerschaft dar, gefolgt in beachtlichem Umfang von Selbständigen (siehe Tabelle 6). Trotz ihres attraktiven Spitzenkandidaten Josef Daxenberger stießen nur vergleichsweise wenige Wähler aus Landwirtschaft und ländlichem Raum neu zu den Grünen.

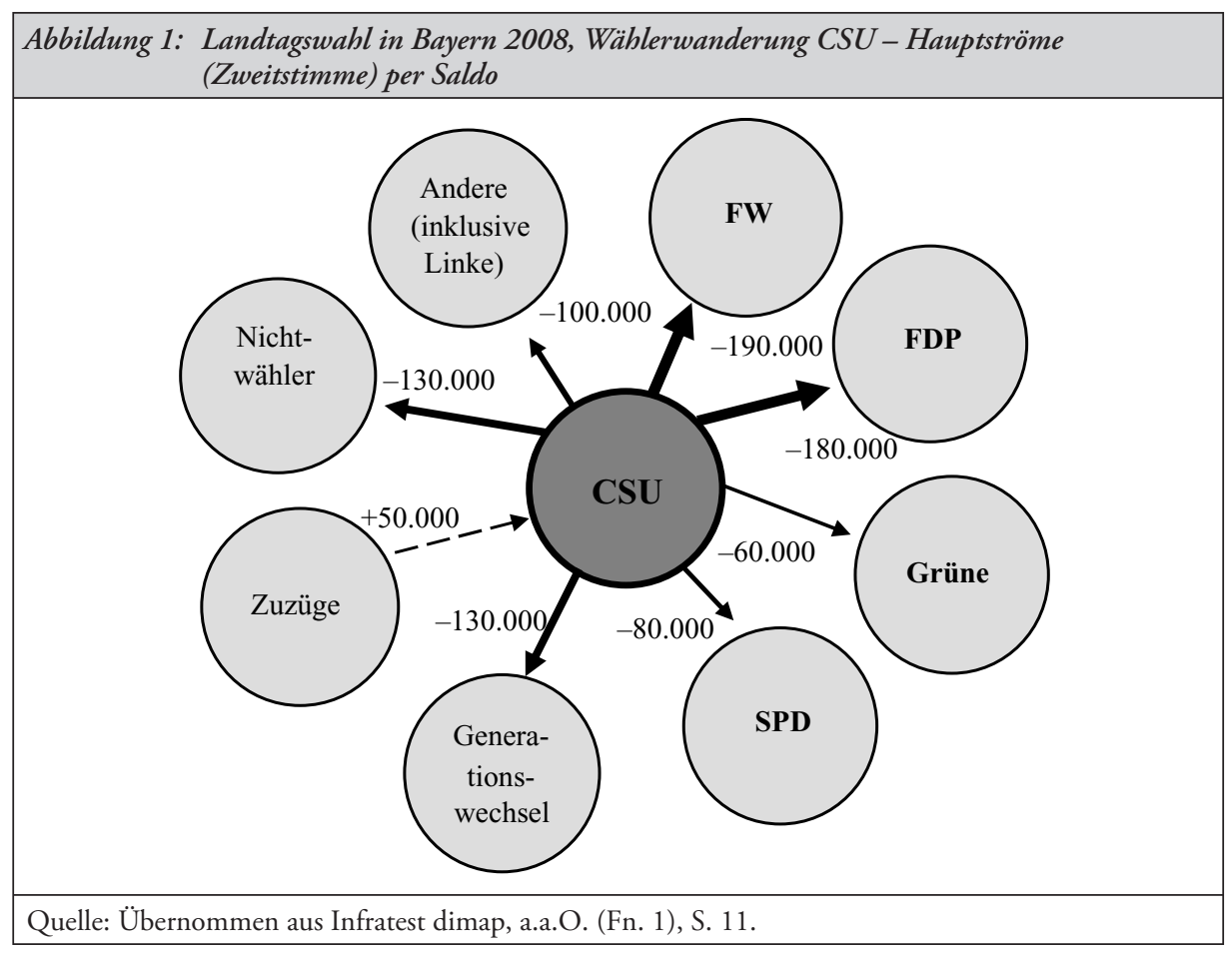

35 Vgl. ebenda, S. 30 f., S. 50 f. 
Auf insgesamt niedrigem Niveau variierten die Stimmenanteile der SPD in verschiedenen Wählergruppen vergleichsweise nur wenig. Dies gilt für den Bildungsgrad wie für die Alterskohorten mit jeweils geringen Varianzen von knapp unter beziehungsweise über 20 Prozent (siehe Tabellen 5 und 6). Deutlichere Unterschiede traten hingegen konfessionell zutage. Zwar waren knapp die Hälfte aller SPD-Wähler katholisch, gemessen an der Gesamtwählerschaft ist dies eine beachtliche Unterrepräsentation von zehn Prozentpunkten, während evangelische und konfessionslose Wähler überrepräsentiert sind. ${ }^{36}$ Mit Blick auf die Berufsstruktur fällt auf, dass die SPD bei Beamten, Angestellten und Rentnern überdurchschnittlich abschnitt, hingegen sich nur 18 Prozent der Arbeiter für sie entschieden, verglichen mit Stimmenanteilen von 41 Prozent für die CSU und immerhin 14 Prozent für die FW. Besonders schmerzlich für die Sozialdemokraten muss dabei sein, dass sie unter den gewerkschaftlich gebundenen Arbeitern und Angestellten starke Verluste erlitten, die überwiegend ins Lager der Linken abwanderten. Im Saldo wechselten 50.000 Stimmen von der SPD zur Linkspartei. ${ }^{37}$

\section{Wählerverhalten im Zeichen strukturierter Vielfalt}

Verglichen mit der vorangegangenen Landtagswahl von 2003 entschieden sich rund zwei Drittel der Wähler für dieselbe Partei. Folgt man den Wanderungsbilanzen von Infratest dimap $^{38}$, gehören von den vormaligen CSU-Wählern, die diesmal nicht oder anders votierten, im Saldo rund 30 Prozent in die Gruppen der Nichtwähler und des Generationenwechsels; rund 45 Prozent von ihnen wechselten lagerintern und 25 Prozent lagerübergreifend (siehe Abbildung 1). Die steigende Volatilität im Elektorat ist einerseits ganz sicher zurückzuführen auf die nachhaltigen Prozesse der Lockerung beziehungsweise Teilauflösung der tradierten sozialen und kulturellen Milieus sowie des doch beachtlicheren Rückgangs der Parteiidentifikation. ${ }^{39}$ Dies signalisiert das Ende der strukturellen Dominanz der Volksparteien auf der Wählerebene ganz generell und jetzt auch der CSU in Bayern. Ihre von vielen fast als „natürlich“, mindestens aber als selbstverständlich angesehenen Wählermehrheiten von 50 Prozent plus x dürften unter „normalen“ Wettbewerbsverhältnissen, und sofern nicht ganz außergewöhnliche Umstände vorliegen, nicht mehr erreichbar sein, denn dazu ist der Prozentsatz der Stammwähler mittlerweile zu deutlich zurückgegangen. ${ }^{40} \mathrm{An}$ -

36 Vgl. FGW, a.a.O. (Fn. 1), 46 f.

37 Nach Infratest dimap, a.a.O. (Fn. 1), S. 10 ff.

38 Vgl. ebenda.

39 Die Parteiidentifikation ist bundesweit seit Ende der 1970er Jahre um circa 15 Prozentpunkte zurückgegangen; zudem hat sich bei den noch gebundenen Wählern die Stärke der Bindung deutlich gelockert. Auch dadurch erhöht sich die Wechselbereitschaft. Von diesen Prozessen der Abnahme fester Parteibindungen sind die Volksparteien und in Bayern damit die CSU am stärksten betroffen.

40 Der Wahlforscher Helmut Jung charakterisierte auf der Veranstaltung der Politischen Akademie Tutzing vom 12. bis 14. Dezember 2008 zur Analyse der Landtagswahl auf der Basis seiner umfänglichen demoskopischen Untersuchungen das Wählerpotential der CSU mit der Formel: 35 + $15+\mathrm{x}$, so dass bei einer Kernwählerschaft von 35 Prozent ein hoher Prozentsatz von nicht oder nur locker gebundenen Wählern jeweils neu gewonnen werden muss. Die Formel macht deutlich, dass ein Ergebnis von 50 Prozent plus x nur mehr unter besonderen Umständen zu erwarten steht. 
dererseits führt dies weder zu Beliebigkeit im Wählerverhalten, noch heißt es, dass sich die Wähler nur noch kurzfristig im Blick auf die Kandidatenalternativen oder sachpolitisch unter Gesichtspunkten „rationaler Wahl“ entscheiden. Vielmehr ist das Wählerverhalten auch weiterhin strukturell vermittelt, wenngleich weniger stabil und vielfältiger, sofern auf der Angebotsseite des Parteienwettbewerbs entsprechende Alternativen vorhanden sind. In Anlehnung an US-amerikanische Wahlforscher ${ }^{41}$ kann man die Bildung der individuellen Wählerentscheidung dabei als Prozess eines komplexen, mehrstufigen Kausalitätszusammenhanges sehen, wobei die Einflussvariablen der nachgeordneten Stufen von den vorherigen beeinflusst werden, die Langfristdeterminanten damit als Filter wirken (siehe Abbildung 2). In Anbetracht der sich lockernden traditionellen Bindungen treten dabei die politisch-ideologischen Grundorientierungen der Wähler zunehmend als Langfristdeterminanten neben und an die Stelle der Parteiidentifikation. Sie sind allerdings weniger stark organisiert, zudem - und anders als die Parteiidentifikation - weniger affektiv, sondern kognitiv auf konkrete Ziele und Programme wie deren Verwirklichung bezogen, die dadurch im Rahmen strukturierter Vielfalt einen höheren Stellenwert für die individuelle Wählerentscheidung gewinnen. ${ }^{42}$

\section{Abbildung 2: Der Kausalitätstrichter in Anlehnung an Miller / Shanks}

Stufe 1: $\quad$ (1) Wirtschafts- und Sozialstruktur

Stufe 2: $\quad$ (2) Parteiidentifikation

(3) Politikbezogene Grundorientierung und ideologische Selbsteinschätzung

Stufe 3: $\quad$ (4) Aktuelle Politikpräferenzen / Themen (Issues)

(5) Einschätzung der allgemeinen Lage

Stufe 4: $\quad$ (6) Retrospektive Leistungsbeurteilung der amtierenden Regierung

Stufe 5:

(7) Einschätzung der Eigenschaften der Kandidaten

Stufe 6:

(8) Prospektive Einschätzung der Kandidaten und Parteien wie der von diesen zu erwartenden Leistungen

Wahlentscheidung

Quelle: Vgl. Warren E. Miller / J. Merrill Shanks, a.a.O. (Fn. 41), S. 192.

Die Wählerwanderungen bei der Landtagswahl von 2008 reflektieren diese Veränderungen. Vormalige CSU-Wähler sind eben nur in relativ geringer Zahl lagerübergreifend zu den parlamentarischen Oppositionsparteien gewechselt; vielmehr sind sie entweder in die Nichtwahl abgewandert oder entlang ihrer politisch-ideologischen Grundüberzeugung zu

41 Siehe Warren E. Miller / J. Merrill Shanks, The New American Voter, Cambridge 1996; vgl. Franz Urban Pappi / Susumu Shikano, Wahl- und Wählerforschung, Baden-Baden 2007, S. 29 ff.; Jörg Broschek / Rainer-Olaf Schultze, Wahlverhalten, a.a.O. (Fn. 2), S. 23 ff.

42 Siehe Rainer-Olaf Schultze, Strukturierte Vielfalt, a.a.O. (Fn. 2). 
Freien Wählern und FDP gewechselt, also den Parteien, deren Grundorientierungen mit ihren eigenen wie derjenigen der Unionspartei am ehesten kompatibel sind (siehe Abbildung 1). Ausgelöst wurde die Abwanderung von der CSU durch die weithin negative retrospektive Leistungsbeurteilung von Partei und Regierung - personell wie sachpolitisch, wobei für viele der abgehobene Handlungsstil des Führungspersonals, der unglückliche Amtswechsel von Stoiber zum Tandem Beckstein/Huber und thematisch die unpopuläre Bildungs- und Schulpolitik ursächlich gewesen sein dürften. In welche Richtung die landesweit zahlenmäßig etwa gleich große Abwanderung erfolgte, ob zur FDP oder zu den Freien Wählern, ist sowohl sozialstrukturell und politisch-ideologisch begründet als insbesondere auch themenbezogen. Die FDP überzeugte mit ihrer rechtsstaatlichen, vor allem aber wirtschaftsliberalen Programmatik Wähler insbesondere aus altem wie neuem Mittelstand. Der Wechsel zu den Freien Wählern beruhte auf deren regional- und lokalpolitisch zentrierten Leistungen der Vergangenheit, die von enttäuschten vormaligen CSU-Wählern in die Zukunft extrapoliert und mit Leistungserwartungen auf dem Felde einer bürgernahen und gerechtigkeitsorientierten Gesellschaftspolitik verknüpft wurden. Dies geht einher mit einer zunehmenden Regionalisierung und Lokalisierung von Politik und politischer Partizipation, die - wohl auch als Reaktion auf die Prozesse der Globalisierung und abgehobener supranationaler Integration - ohnehin seit geraumer Zeit zu beobachten sind. Lokale wie regionale Themen und Reformerwartungen wie -befürchtungen, etwa im Bereich der Umwelt oder der Entwicklung des ländlichen Raumes, beispielsweise in der Frage genveränderter Landwirtschaft, bei Megaprojekten des Infrastrukturausbaus wie in Sachen Donaukanalisierung und Flughafenausbau, spielen in der Wählerentscheidung und nicht nur auf kommunaler Ebene eine immer wichtigere Rolle. Dies führt zur Pluralisierung des Wählerverhaltens, von dem die kleineren Parteien - und keineswegs nur die vermeintliche „AntiPartei“ der Freien Wähler - profitieren, die themenzentrierter und auch authentischer ihre Programmatik vertreten können, während „Großparteien“ wie die CSU gerade wegen ihres „Omnibus-Charakters“ immer weniger in der Lage sind, die verschiedenen und zunehmend fragmentierteren Wählersegmente entlang weniger diffuser oder symbolischer Globalthemen zu integrieren.

\section{Regierungsbildung und Oppositionsformierung}

Unter dem Eindruck ihrer Wahlniederlage und unter dem Zeitdruck kurzfristiger Koalitions- und Regierungsbildung ${ }^{43}$ tauschte die Union innerhalb von nur einer Woche ihr Führungspersonal aus. Zu groß waren die Erschütterungen in der Partei, tatkräftig befördert vom Amtsvorgänger Stoiber und dem oberbayerischen Bezirksverband, als dass das Tandem Beckstein/Huber hätte weiter amtieren können. Zunächst trat Huber als Parteivorsitzender zurück und musste Horst Seehofer das Feld überlassen, der sodann auch Beckstein als Ministerpräsident beerbte ${ }^{44}$, so dass Partei- und Regierungsspitze neuerlich in einer

43 Laut bayerischer Verfassung hat die konstituierende Sitzung des Landtags spätestens am 22. Tag nach der Wahl (Art. 12), die Wahl des Ministerpräsidenten sodann innerhalb einer Woche zu erfolgen (Art. 44).

44 Und dies obwohl bekanntlich eine klare Mehrheit der Wähler in den Umfragen zur Kandidatenalternative Beckstein und nicht den Herausforderer Maget als Ministerpräsidenten präferiert hatte, vgl. Infratest dimap, a.a.O. (Fn. 1), S. 36 ff. 
Hand vereinigt sind. Als Koalitionspartner kamen für die CSU rechnerisch sowohl die FDP (mit zusammen 108 von 187 Sitzen im Landtag) als auch die Freien Wähler (mit zusammen 113 Sitzen) infrage; sachpolitisch wie vor allem parteitaktisch kam indessen nur die schwarz-gelbe Koalition aus CSU und FDP in Betracht, deren soziale wie regionale Wählerklientele - und anders als im Falle der Freien Wähler - sich eher ergänzen und die trotz aller Differenzen in der Rechts- und Bildungspolitik, in der Wirtschafts-, Steuer- und Haushaltspolitik nah beieinander sind. Infolgedessen einigte man sich im Koalitionsvertrag schnell und wechselseitig auf den umstrittenen Feldern auf Kompromisse, wobei die Liberalen in der Schulpolitik, die Union vor allem in der Rechts- und Innenpolitik Abstriche von ihren jeweiligen Programmpositionen hinnehmen mussten.

Personell setzte Seehofer eine deutliche Verjüngung des Kabinetts durch; allerdings musste er bei der Regierungsbildung nicht nur auf den liberalen Koalitionspartner, sondern erneut auch auf den innerparteilichen CSU-Regionalproporz Rücksicht nehmen. An die FDP fielen zwei Ressorts: Minister für Wirtschaft, Infrastruktur, Verkehr und Technologie (und Stellvertretender Ministerpräsident) wurde Martin Zeil. Wolfgang Heubisch übernahm von Thomas Goppel das Ministerium für Wissenschaft, Forschung und Kunst. Die Riege der CSU-Minister im Kabinett wird angeführt von dem oberbayerischen Bezirksvorsitzenden Siegfried Schneider, vormals wenig erfolgreich im Kultusministerium, als Staatsminister und Leiter der Staatskanzlei. Ihre Ressorts behielten Innenminister Joachim Herrmann und Beate Merk als Justizministerin, deren Geschäftsbereich um den Verbraucherschutz erweitert wurde. Dem regionalpolitischen Proporz geschuldet, profitierte von der parteiinternen Rotation auch Emilia Müller, von Oktober 2007 bis Oktober 2008 Ministerin für Wirtschaft, Infrastruktur, Verkehr und Technologie, die erneut die Bundes- und Europaangelegenheiten übernahm, für die sie bereits in der letzten Regierung Stoiber von November 2005 bis September 2007 verantwortlich gewesen war, während Markus Söder, vormals Generalsekretär der Partei und Bundes- und Europaminister, in das einflussreiche Ressort für Umwelt und Gesundheit wechselte. Zum Kultusminister avancierte Ludwig Spaenle, bis dato Vorsitzender des Landtagsausschusses für Hochschule, Forschung und Kultur. Die Nachfolge des bisherigen Landwirtschaftsministers Josef Miller tritt Helmut Brunner an, von 2003 bis 2008 Vorsitzender des entsprechenden Landtagsausschusses. Die als Generalsekretärin gescheiterte Wahlkampfmanagerin Christine Haderthauer wird neue Ministerin für Arbeit und Sozialordnung, Familie und Frauen und löst dort Christa Stewens ab. ${ }^{45}$ Starker Mann im Kabinett nach Regierungschef Seehofer dürfte Georg Fahrenschon werden, der als Nachfolger von Erwin Huber das strategisch wichtige Finanzressort übernimmt.

Fraktionsvorsitzender der CSU im Landtag bleibt Georg Schmid, dessen Amtsführung allerdings nicht unumstritten ist und dessen Verhältnis zu Parteichef und Ministerpräsident Seehofer nicht ungetrübt zu sein scheint. Auch Franz Maget wurde als Fraktionsvorsitzender für die SPD bestätigt und führt nun schon seit dem Jahr 2000 die Sozialdemokraten im Maximilianeum, zunächst bis zur Mitte der Wahlperiode, da mit einer neuerlichen Spitzenkandidatur seinerseits nicht zu rechnen sein dürfte. Als Fraktionsführer der Grünen amtieren der vormalige Landesvorsitzende Sepp Daxenberger und die ehemalige Fraktionsspre-

45 Als Staatssekretäre mit Kabinettsrang amtieren: Bernd Weiß (CSU - Inneres), Marcel Huber (CSU - Unterricht und Kultus), Franz Pschierer (CSU - Finanzen), Melanie Huml (CSU - Umwelt und Gesundheit), Markus Sackmann (CSU - Arbeit und Sozialordnung, Familie und Frauen) und Katja Hessel (FDP - Wirtschaft, Infrastruktur, Verkehr und Technologie). 


\begin{tabular}{|l|l|}
\hline Tabelle 7: Das Kabinett nach der bayerischen Landtagswahl vom 28. September 2008 \\
\hline Ministerpräsident & Horst Seehofer (CSU) \\
\hline Leiter der Staatskanzlei & Siegfried Schneider (CSU) \\
\hline Staatsministerin für Bundes- und Europaangelegenheiten & Emilia Müller (CSU) \\
\hline Innenminister & Joachim Herrmann (CSU) \\
\hline Justiz- und Verbraucherschutzministerin & Beate Merk (CSU) \\
\hline Wissenschafts-, Kunst- und Forschungsminister & Wolfgang Heubisch (FDP) \\
\hline Unterrichts- und Kultusminister & Ludwig Spaenle (CSU) \\
\hline Finanzminister & Georg Fahrenschon $(\mathrm{CSU})$ \\
\hline Wirtschafts-, Infrastruktur-, Verkehrs- und Technologieminister & Martin Zeil (FDP) \\
\hline Umwelt- und Gesundheitsminister & Markus Söder (CSU) \\
\hline Ernährungs-, Landwirtschafts- und Forstminister & Helmut Brunner (CSU) \\
\hline Arbeits-, Sozial-, Familien- und Frauenministerin & Christine Haderthauer $(\mathrm{CSU})$ \\
\hline
\end{tabular}

cherin Margarete Bause, während der bisherige Ko-Sprecher Sepp Dürr ins zweite Glied rückte. Der Fraktion der Freien Wähler steht Hubert Aiwanger und der FDP-Fraktion Thomas Hacker vor.

Die Koalitionsregierung aus Union und Liberalen verfügt im Landtag über eine solide Mehrheit. Ihr Start ist zum einen wesentlich bestimmt von der bundespolitischen Aufmerksamkeit, die Horst Seehofer als CSU-Parteivorsitzendem entgegengebracht wird, zum anderen landespolitisch durch den Versuch, auf einigen Politikfeldern neue Akzente zu setzen. Dies gilt für die Bildungs-, Schul- und Hochschulpolitik wie für den Bereich der Umwelt- und Infrastrukturpolitik, wo man bemüht ist, durch Umorientierung (etwa auch beim Donauausbau) auf die Bürgerinteressen zuzugehen. Ob der mit der Regierungserklärung vom 10. Dezember 2008 „Zukunft gemeinsam gewinnen - mit Mut und Selbstvertrauen " 46 versprochene Neuanfang tatsächlich gelingt, wird man abwarten müssen. Dies betrifft den Regierungsstil und vor allem die Frage, ob die Zusammenarbeit der beiden Koalitionspartner auf Dauer reibungslos vonstatten gehen wird. Neben der Innenund Rechtspolitik werden insbesondere die Wirtschafts- und Finanzpolitik hierfür den Testfall liefern, zumal die Handlungsspielräume für Politikinnovationen durch die allgemeine Finanz- und Wirtschaftskrise sowie insbesondere durch die zehn Milliarden Euro Belastung des Landeshaushaltes aus dem Fiasko der Bayern LB zukünftig doch stärker eingeengt sein werden. ${ }^{47}$

46 Die Leitlinien der Regierungserklärung lauten „Geborgenheit und soziale Sicherheit“, „gute Bildung und Teilhabe für alle“, „Nachhaltigkeit und Generationengerechtigkeit“, „persönliche und wirtschaftliche Freiheit sowie verlässlicher staatlicher Schutz“. Die Leitlinien finden sich auch in der Koalitionsvereinbarung zwischen CSU und FDP für den Zeitraum 2008 bis 2013, der Text der Regierungserklärung unter: http://www.bayern.de/Regierungserklaerungen-.1290.10126770/ index.htm, Abruf am 15. Dezember 2008.

$47 \mathrm{Ob}$ das Wahlergebnis für die CSU noch negativer ausgefallen wäre, wenn das Ausmaß der finanziellen Belastung vor dem Wahltag bekannt gewesen wäre, ist eine offene und - weil kontrafaktisch - kaum verlässlich zu beantwortende Frage. 


\section{Zusammenfassung}

(1) Die Wahlbeteiligung erreichte das bei Landtagswahlen gegenwärtig übliche Maß von knapp unter 60 Prozent. Der Durchschnittswert darf allerdings die doch deutlichen Differenzen in den Partizipationsraten nicht überdecken. Denn von den Wählern unter 25 Jahre beteiligten sich nur magere 40 Prozent.

(2) Die allgemein zu beobachtende Pluralisierung des Parteiensystems führt jetzt auch im Bayerischen Landtag zu einem Fünf-Fraktionen-Parlament des „gemäßigten Pluralismus “48. Union und Sozialdemokraten kommen zusammen nur noch auf 62 Prozent der Stimmen, während die drei kleineren Parlamentsparteien 30 Prozent auf sich vereinigen. Die Abwanderung von den „Großparteien“ entspricht auch im Umfang den bundesweiten Trends; sie dürften mittelfristig stabil sein.

(3) Die dramatischen Stimmenverluste der CSU von 17,3 Prozentpunkten sind zu einem Gutteil kurzfristig situativen und konjunkturellen Faktoren geschuldet - nämlich vor allem einem Regierungsstil, der geprägt war von der Arroganz der Macht einer seit 46 Jahren alleinregierenden Partei. Hinzu kamen die Turbulenzen und die Agonie eines fast einjährigen Amtswechsels von Edmund Stoiber zum Tandem Beckstein/Huber sowie eine sachpolitische Agenda während der Wahlperiode, die an den Interessen und Befindlichkeiten großer Teile der Wählerschaft vorbeiging. Dies betraf eine Vielzahl von Politikfeldern und nicht allein die Schul- und Bildungspolitik, und es veranlasste viele Wähler, ihre negative Leistungsbewertung in die Zukunft zu extrapolieren und zur Grundlage ihrer (retrospektiven) Wahlentscheidung zu machen, zumal Beckstein und Huber es nicht vermochten, die Ära Stoiber hinter sich zu lassen.

(4) Thematisch war diese Landtagswahl primär von der Landespolitik bestimmt. Für zwei Drittel der Wähler waren die landespolitischen Probleme wichtiger als bundespolitische Themen und Einflüsse. ${ }^{49}$ Pendlerpauschale und Steuerentlastung, strittig in der Berliner Großen Koalition und zwischen den Schwesterparteien, mit denen die CSU im Wahlkampf vergeblich zu mobilisieren versuchte, rangierten unter den wichtigsten Themen nur an fünfter Stelle. ${ }^{50}$ Sie mögen für Parteiwechsler zur FDP eine gewisse Bedeutung gehabt haben, spielten insgesamt aber nur eine untergeordnete Rolle.

(5) Die herausgehobene Bedeutung der Landespolitik für die Wählerentscheidung resultierte auch daraus, dass die CSU ihre institutionelle Doppelrolle als bayerische „Staats- und Ordnungspartei“ (Alf Mintzel) und als eigenständige, im Aktionsbündnis mit der CDU zusammengeschlossene Bundespartei, die in der Vergangenheit ihre Stärke in Land wie Bund wechselweise begründete, kaum noch erfolgreich wahrnehmen konnte. Denn nach Bildung der Großen Koalition und dem Rückzug Edmund Stoibers nach München verlor die CSU als kleinster Partner der Berliner Drei-Parteien-Koalition stark an bundespolitischem Einfluss. Damit entfiel für viele bayerisch gesinnte Traditionswähler ein zentrales Wahlmotiv, und die landespolitischen Alternativangebote der kleineren Mitbewerber gewannen an Attraktivität. Insofern überrascht es nicht, dass die Führung von

48 Siehe Giovanni Sartori, Parties and Party Systems, Cambridge 1976; Klaus von Beyme, Parteien im Wandel, Wiesbaden 2000, S. $158 \mathrm{ff}$.

49 Vgl. FGW, a.a.O. (Fn. 1), S. 20.

50 Vgl. Infratest dimap, a.a.O. (Fn. 1). 
Partei und Regierung jetzt neuerlich in den Händen eines ausgewiesenen Bundespolitikers liegt und sich Horst Seehofer nach Kräften darum bemüht, die traditionelle Doppelrolle der CSU wieder erfolgreich zu spielen.

(6) Die Verluste der CSU sind zugleich aber auch gesellschaftsstrukturell begründet und Konsequenz der tief greifenden Prozesse der ökonomischen Modernisierung und des sozialen Wandels. Zur Erosion der Milieus und der Auflösung der traditionellen soziokulturellen und parteilichen Bindungen wie zum damit einhergehenden Wertewandel haben die CSU-Regierungen der Vergangenheit durch ihre erfolgreiche Politik der wirtschaftlich-technologischen Entwicklung des Landes selbst beigetragen. Mit zeitlicher Verzögerung trifft die Partei jetzt auch in Bayern eine Entwicklung, die CDU und SPD in ihren Hochburgen schon früher zu spüren bekommen haben. ${ }^{51}$ Negativ betroffen ist die Volkspartei CSU zudem von der zunehmenden Fragmentierung der Wählerschaft wie auch von der Regionalisierung und Lokalisierung der Politik. Daraus folgt eine Vervielfältigung wahlentscheidender Themen, die nur noch schwer auf einen gemeinsamen Nenner gebracht werden können und die fragmentierten Wählerschaften veranlassen, sich nach alternativen Wahlangeboten umzusehen, so dass die Wechselbereitschaft auch aus diesem Grunde deutlich gestiegen ist.

(7) Die Volatilität ist indes nicht beliebig. Die Bewertung von Kandidatenalternativen, Parteien und Themenkompetenzen bleibt gebunden an die Langfristdeterminanten, vor allem an die politisch-ideologischen Grundüberzeugungen, und führt zu strukturierter Vielfalt in Parteiensystem und Wählerverhalten. Von der Wechselstimmung profitierten denn auch - wahlsoziologisch durchaus erwartbar - die kleinen Parteien des „bürgerlichen Lagers“, Liberale und Freie Wähler, nicht jedoch die bisherige Parlamentsopposition aus Sozialdemokraten und Grünen.

(8) Mit dem Erdrutsch-Ergebnis der Landtagswahl vom 28. September 2008 hat sich die politische Landschaft in Bayern stark und nachhaltig verändert. Der Mythos der CSU als „natürlicher“ bayerischer Staatspartei ist gebrochen. An die Stelle der Alleinregierung ist die Koalitionslogik getreten, die die Mandats- und Funktionsträger der Partei erst noch lernen müssen. Allerdings konnte die CSU in dem asymmetrisch von einer Großpartei und mehreren mittelgroßen Parteien strukturierten Parteiensystem ihre führende Position behaupten. Zudem sind die Oppositionsparteien nur wenig schlagkräftig aufgestellt. Dies gilt weniger für die „Premiumopposition“ von Bündnis 90/Die Grünen als für die Sozialdemokraten und Freien Wähler. Letztere müssen sich erst noch in ihre Rolle als landes- und gegebenenfalls auch als bundes- und europaweit agierende Partei hineinfinden und ringen nicht von ungefähr mit der Überwindung ihres „AntiParteien“-Selbstverständnisses. Insofern wird die weitere Entwicklung der bayerischen Landespolitik nicht zuletzt von der Wahl zum Europäischen Parlament Anfang Juni 2009 beeinflusst. Diese Wahl wird zum Testfall nicht nur für die CSU, sondern insbesondere für die Freien Wähler, wobei die kurz- wie mittelfristigen politischen Aussichten beider davon abhängen, ob die Freien Wähler kandidieren und welches Ergebnis sie erzielen.

51 Heinrich Oberreuter veranlasste dieser Zusammenhang auf der schon genannten Tagung in Tutzing zu dem pointierten, aber durchaus treffenden Aphorismus: „Die Modernisierung frisst ihre Eltern." 